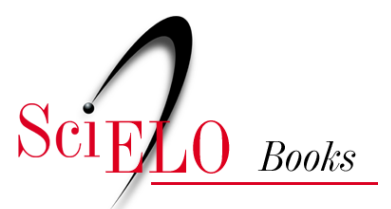

\title{
6. A Abrasco e a Experiência da Graduação em Saúde Coletiva
}

\author{
Isabela Pinto \\ Jairnilson Paim
}

\section{SciELO Books / SciELO Livros / SciELO Libros}

PINTO, I., and PAIM, J. A Abrasco e a Experiência da Graduação em Saúde Coletiva. In: LIMA, N.T., SANTANA, J.P., and PAIVA, C.H.A., orgs. Saúde coletiva: a Abrasco em 35 anos de história [online]. Rio de Janeiro: editora FIOCRUZ, 2015, pp. 137-150. ISBN: 978-85-7541-590-0. Available from: doi: $10.7476 / 9788575415900.0008$. Also available in ePUB from: http://books.scielo.org/id/q4gzb/epub/lima-9788575415900.epub.

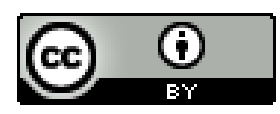

All the contents of this work, except where otherwise noted, is licensed under a Creative Commons Attribution 4.0 International license.

Todo o conteúdo deste trabalho, exceto quando houver ressalva, é publicado sob a licença Creative Commons Atribição 4.0. 


\title{
A Abrasco e a Experiência da Graduação em SaÚde Coletiva
}

Isabela Pinto

Jairnilson Paim

\begin{abstract}
Alguns estudos têm discutido a saúde coletiva (SC) no âmbito de profissionalização, apontando fundamentos teóricos, sociais e ético-políticos para uma formação em nível de graduação (Padilha, 1997; Bosi, Raggio \& Medronho, 2001; Teixeira, 2003; Elias, 2003; Paim, 2006; Bosi \& Paim, 2009). Outros buscam recuperar a trajetória da criação dos cursos de graduação em saúde coletiva (CGSC) e a sua implantação em universidades brasileiras (Ruela, 2013; Santos, 2014).
\end{abstract}

Cabe lembrar que uma das primeiras iniciativas da então Associação Brasileira de PósGraduação em Saúde Coletiva (Abrasco) foi organizar, em 1983, uma reunião nacional para discutir o ensino da SC na graduação. Apesar de não haver o propósito de implantar CGSC naquela oportunidade, tal iniciativa indicava uma preocupação da entidade com a formação profissional na área, como contribuição para o projeto da Reforma Sanitária brasileira (RSB). Os participantes do evento recomendarama organização deum núcleo degraduaçãonoâmbito da Abrasco e uma articulação com a Associação Brasileira de Educação Médica (Abem) e com os departamentos de medicina preventiva e social (Abrasco, 1984).

A ideia de uma graduação em SC vem sendo debatida no Brasil, desde o fim da década de 1970, em instituições de ensino, como a Escola Nacional de Saúde Pública Sergio Arouca da Fundação Oswaldo Cruz, ao examinar alternativas com base nas avaliações dos cursos básicos regionalizados de saúde pública (CBRSP), envolvendo professores como Sérgio Arouca, Mario Hamilton, Arlindo Fábio e Paulo Buss, entre outros (Santos, 2014). Apesar de não concretizada naquele momento, essa ideia foi compartilhada com lideranças acadêmicas de universidades brasileiras e debatida, em 1993, noCongresso da RedeUnida em 
São Paulo e em seminário de comemoração dos 20 anos do Programa de Pós-Graduação em Saúde Coletiva da Universidade Federal da Bahia (Ufba), em Salvador. Assim, desde essaépoca era indicada a necessidade de antecipação da formação do chamado sanitarista, até então realizada nos cursos de pós-graduação, a ponto de essa proposta integrar os documentos de criação do Instituto de Saúde Coletiva (ISC) da Ufba em 1994.

Considerando o desenvolvimento da SC nesse período, torna-se relevante apresentar os fundamentos e a evolução dessa proposta para estudantes, profissionais da saúde, gestores e demais interessados na consolidação do Sistema Único de Saúde(SUS), no momento em que a Associação Brasileira de Saúde Coletiva (Abrasco) comemora 35 anos de existência. Este capítulo tem como objetivo analisar a conformação da graduação em SC, discutindo seus antecedentes, o processo de implantação dos distintos cursos e o protagonismo da Abrasco nessa construção.

\section{AnTECEDENTES}

A partir da ideia de uma graduação em SC esboçada nas iniciativas mencionadas, foi elaborado um texto inicial para discussão interna no ISC/Ufba (Padilha, 1997). Reconheciase, naquela oportunidade, que o profissional da saúde dedicado aos problemas coletivos que afligem as comunidades tem sido reconhecido como sanitarista ou salubrista. Mesmo considerando-se que tal denominação pudesse ser redefinida diante da crise da saúde pública institucionalizada e da construção do campo da SC, estabeleciam-se convergências, diálogos e identidades entre escolas de saúde pública e institutos de saúde coletiva ou assemelhados no Brasil. Admitia-se que um perfil geral desse profissional contemplaria o conhecimento de doenças, agravos, riscos e determinantes de saúde, assim como as respostas sociais e institucionais a eles, reservando a assistência aos doentes para os tradicionalmentegraduados para atendê-los. Argumentava-se ainda no texto que a formação desse profissional na graduação prescindiria, atécerto ponto, dos conhecimentos clínicose terapêuticosespecíficos, voltados para o atendimento individual. Sua habilitação, porém, deveria capacitá-lo a cooperar com as equipes de saúde (Padilha, 1997).

A transformação dessa ideia numa proposta preliminar no âmbito do ISC/Ufba ocorreu mediante entendimentos com a direção da então Secretaria de Políticas de Saúde do Ministério da Saúde. No cenário nacional, cabe ressaltar que em 2001 a Universidade Estadual do Rio Grande do Sul (Uergs) lançou, após debate em todo o estado, um conjunto de cursos degraduação, definidos deacordo com prioridades regionais. Dentreas prioridades, emergiua SC, com destaque para as demandas na gestão do SUS, resultando na criação do bacharelado em administração de sistemas e serviços de saúde (Ceccim, 2002). Simultaneamente, foi apresentado um projeto de criação de um curso de graduação em SC na Universidade Federal do Rio de Janeiro, contando com o apoio do reitor recém-eleito. 
Em setembro de 2002, o ISC/Ufba organizou uma oficina de trabalho em Salvador, promovida pelo Ministério da Saúde, pela Abrasco e pela Organização Pan-Americana da Saúde (Opas) - escritório do Brasil -, reunindo professores e dirigentes da Ufba, além de representantes de universidades e das referidas instituições. Para esse evento, foi elaborado um texto de referência com o objetivo de discutir o objeto e a prática da SC, analisando a pertinência da formação de um novo profissional no âmbito da graduação (Paim, 2006).

Ressaltava-se no documento o desenvolvimento teórico-conceitual da área de SC e a experiência acumulada no processo de reforma do sistema de saúde brasileiro, especialmente as tendências de mudança do modelo de atenção à saúde e as demandas do mercado de trabalho no setor. Considerava-se que a SC dispunha de maturidade teórica, metodológica e operativa para enumerar competências e articular valores que orientassem a constituição de novos sujeitos comprometidos com a defesa da vida e da saúde do público. Apontavam-se a crise da saúde pública e a busca de novos paradigmas estimulada pela Opas, Organização Mundial da Saúde (OMS), Academia de Medicina dos Estados Unidos e Associação LatinoAmericana e do Caribe de Educação em Saúde Pública (Alaesp).

Naquela oportunidade, foram discutidas novas ideias e reflexões elaboradas por experts convidados pela Opas para debater a crise e certas propostas alternativas (Paho, 1992). Argumentava-se, enfim, que a saúde pública e a SC não constituem especialidade médica (Macedo, 1992; Belisário, 1995). Ainda que a SC componha a área da saúde, suas conexões são mais amplas e profundas com as ciências humanas e sociais, a exemplo de economia, administração, comunicação social e marketing, pedagogia e direito, entre outras. Nessa perspectiva, o texto sublinhava a recomendação da $1^{\text {a C} C o n f e r e ̂ n c i a ~ P a n-A m e r i c a n a ~ d e ~}$ Educação em Saúde Pública (Alaesp, 1994) para queas instituições formadoras desses agentes daspráticasdesaúdepassassemporumprocessodeindependizaçãodasfaculdadesdemedicina, estabelecendo redes com outras unidades acadêmicas visando à interdisciplinaridade e à transdisciplinaridade.

Portanto, buscava-se no documento subsidiar a discussão dos participantes sobre a pertinência, a oportunidade ea viabilidade da proposta. Assim, nesse $1{ }^{\circ}$ Seminário Graduação em Saúde Coletiva: pertinência e possibilidades, diversos participantes reconheceram a viabilidade, a pertinência e a necessidade de um curso de SCem nível de graduação (ISC/Ufba, 2002), ao tempo que recomendaram ampliar as discussões sobre tal proposta. As reflexões e proposições desseevento conduziramà conclusão de queera oportuno avançarna elaboração do projeto político-pedagógico do curso, bem como ampliar a análise de viabilidade da sua implantação, não só na Ufba como também em outras instituições de ensino superior no país (Teixeira, 2003). Dentre as recomendações, destacava-se a necessidade de ampliação do debate sobre o tema, contando com a decidida colaboração da Abrasco no $7^{\circ}$ Congresso Brasileiro de Saúde Coletiva de 2003.

Antes mesmo desse congresso, o tema foi debatido no Fórum Social Mundial em Porto Alegre, em janeiro de 2003, numa mesa-redonda promovida pela Uergs (Santos, 2014). 
Depois, como atividade pré-congresso do referido Abrascão, realizou-se uma segunda oficina de trabalho sobre graduação em saúde coletiva. Na programação oficial do evento, foiapresentado o painel “É tempo de termos uma graduação em saúde coletiva?", contando com uma plateia de cerca de oitocentos participantes (Bosi \& Paim, 2010). As controvérsias e o intenso debate nesse painel derivaram para uma polarização entre os que defendiam a SC como parte da formação das profissões da saúde (Almeida, 2003) e os que argumentavam a favor de uma nova graduação, sem prejuízo da formação em SC dos demais profissionais (Paim, 2003).

As recomendações da oficina desse Abrascão reiteraram a indicação de se ampliar o debate. No entanto, nem a Abrasco nem o Ministério da Saúde viabilizaram tais desdobramentos naquela época, ainda que algumas universidades envidassem esforços para a institucionalização da proposta em instâncias e colegiados superiores (Bosi \& Paim, 2010). Portanto, até julho de 2003 a proposta contou com a simpatia e o apoio da direção da Abrasco. Daí em diante, até 2009, o tema deixou de ter relevo na agenda da entidade. Nesse período, algumas propostas de cursos foram encaminhadas para a análise de instâncias colegiadas de universidades públicas, como no caso da Ufba, com a tentativa de implantação do projeto no campus avançado do município de Vitória da Conquista em 2005 (Santos, 2014). Diante do recuo da Abrasco e do Ministério da Saúde, algumas tentativas de articulação com o Conselho Nacional de Secretarias Municipais de Saúde (Conasems) e o Conselho Nacional de Secretários de Saúde (Conass) foram efetuadas por lideranças dos próprios cursos.

\section{Reuni, a Implantação dos CGSC, a Organização de Instâncias Coletivas e o Protagonismo Estudantil e da Abrasco}

O Programa de Apoio a Planos de Reestruturação e Expansão das Universidades Federais (Reuni) indicava o interesse governamental e a possibilidade de redirecionamento de investimentos públicos para a ampliação da oferta e qualidade do ensino superior. Esse programa tinha como objetivo "criar condições para a ampliação do acesso e permanência na educação superior, no nível de graduação, pelo melhor aproveitamento da estrutura física e de recursos humanos existentes nas universidades federais" (Brasil, 2007). Assim, a proposta dos CGSC aproveitou essa janela de oportunidade, possibilitando o aparecimento de várias iniciativas nessa perspectiva em um conjunto de universidades federais do Brasil (Paim \& Pinto, 2013).

Desse modo, o decreto presidencial n. 6.096, de 24 de abril de 2007 (Brasil, 2007), que criou o Reuni, permitiu que as universidades examinassem a possibilidade da abertura de novos cursos de graduação, ampliando as vagas para o ensino superior e as perspectivas de formação universitária, movimento no qual se insere a criação dos CGSC. A partir do Reuni, o debate deslocou-se da pertinência (o porquê da graduação em SC para o como implantare implementar os CGSC (Bosi \& Paim, 2010). 
Outras iniciativas de articulação de instituições proponentes dos CGSC foram empreendidas via rede, com reuniões em Belo Horizonte e Salvador, a exemplo do Encontro Nacional sobre a Implantação do Curso de Graduação em Saúde Coletiva, promovido pela Ufba, com o apoio do Ministério da Saúde e da Opas, em agosto de 2008. O ministério, que tinha excluído os CGSC da sua agenda desde 2003, colaborou na realização desse encontro, sinalizando uma nova posição política diante do Reuni e da atuação do Ministério da Educação.

No caso da Abrasco, o apoio do seu Grupo Temático (GT) Trabalho e Educação na Saúde foi importante no acompanhamento do processo de implantação dos cursos, contribuindo para que, no $9^{\circ}$ Congresso Brasileiro de Saúde Coletiva em Recife (2009), o tema fosse reintroduzido na agenda da entidade. Nessa oportunidade, foram realizadas a 4a Reunião Nacional dos CGSC e a Oficina de Trabalho Graduação em Saúde Coletiva, promovida pelo mencionando GT, além de uma mesa-redonda sobreotema, coma apreciação positiva dos cursos recém-implantados. Do mesmo modo, o discurso de posse do novo presidente da entidade defendia explicitamente os CGSC, enquanto a Abrasco assumia o compromisso de criar o Fórum de Graduação em Saúde Coletiva (FGSC), composto por um fórum de coordenadores e um fórum ampliado, com representantes de gestores do SUS e de movimentos sociais.

O FGSC foi criado no ano seguinte, durante o $1^{\circ}$ Congresso Brasileiro de Política, Planejamento e Gestão em Saúde, tendo a sua primeira reunião em Brasília em novembro de 2010, na mesma oportunidade da realização da $5^{\mathrm{a}}$ Reunião Nacional dos CGSC. A 2a Reunião Nacional do FGSC e a $6^{\text {a }}$ Reunião Nacional dos CGSC ocorreram em São Paulo, durante o $5^{\circ}$ Congresso Brasileiro de Ciências Sociais em Saúde da Abrasco. Já a 3a a a $4^{a}$ e a $5^{a}$ Reuniões do FGSC aconteceram, respectivamente, no $8^{\circ}$ Congresso Brasileiro de Epidemiologia (São Paulo, em 2011), no $10^{\circ}$ Congresso Internacional da Rede Unida (Rio de Janeiro, em 2012) e no $10^{\circ}$ Congresso Brasileiro de Saúde Coletiva (Porto Alegre, em 2012). Assim, todos os congressos da Abrasco vêm possibilitando reuniões nacionais. Além disso, o colegiado gestor do FGSC tem realizado reuniões por teleconferência (Ruela, 2013).

Em abril de 2011, diante da mobilização dos estudantes dos CGSC e de outros segmentos da SC, foi aprovada a mudança do nome da Abrasco para Associação Brasileira de Saúde Coletiva, enquanto as instituições de educação superior que compõem o Fórum de Coordenadores de Graduação em Saúde Coletiva foram reconhecidas como associados institucionais, e os estudantes dos CGSC como associados individuais. Assim, no período 2009-2014, a questão da graduação em SC foi incorporada na agenda política da Abrasco, quando a associação participou ativamente no processo de formulação e implementação da proposta.

No caso do protagonismo estudantil, o $9^{\circ}$ Abrascão, em Recife, também pode ser consideradoum marco, quandoserealizoua $1^{\mathrm{a}}$ Assembleia Geral dos Estudantes. A2 ${ }^{\mathrm{a}} \mathrm{Assembleia,}$ 
em Salvador (2010), contou com a participação de mais de cem estudantes, e no ano seguinte foi realizado o $1^{\circ}$ Encontro Nacional de Estudantes de Saúde Coletiva (Enesc), oportunidade em que se criou a Coordenação Nacional dos Estudantes de Saúde Coletiva (Conesc). O $2^{\circ}$ Enesc ocorreu em 2012 (Esteio, RS), antecedendo o 10 Abrascão. Nesse evento foram realizadas mesas-redondas para discussão dos CGSC, a exemplo da intitulada "Graduação em saúde coletiva: formação, carreira e profissão", e apresentados diversos trabalhos (Ruela, 2013).

Como desdobramento dessas discussões, foram propostos referenciais curriculares, objeto de debate em novembro de 2013, durante o Congresso de Ciências Sociais da Abrasco no Rio de Janeiro (Santos, 2014), para fundamentarem a elaboração das diretrizes curriculares para os CGSC. Diante das iniciativas da Abrasco e da organização dos estudantes mediante centros e diretórios acadêmicos, encontros nacionais, participação em fóruns da área e de uma instância nacional (Conesc), o debate sobre os CGSC estimulou novas investigações no sentido de responder às perguntas por que, para que e para quem o curso (Ruela, 2013).

\section{Caracterização dos Cursos no InÍCIO da Implantação}

O processo de implantação e monitoramento dos CGSC em diferentes regiões do Brasil foi objeto de investigação realizada pelo GT de Trabalho e Educação na Saúde da Abrasco. ${ }^{1}$ Os resultados do estudo constituíram uma base importante para subsidiar análises de temas que integram o cotidiano dos programas de graduação em SC, considerando os interesses e perspectivas dos profissionais da área, dos membros do GT e dos coordenadores e docentes dos referidos cursos (Abrasco, 2010).

Esse estudo exploratório realizado em 2010 efetuou uma análise documental dos projetos político-pedagógicos (PPPs) e realizou entrevistas com os coordenadores dos cursos em funcionamento naquele ano - universidades federais de Minas Gerais, Bahia, Rio de Janeiro, Rio Grande do Norte, Acre, Mato Grosso, Paraná (litoral) e Universidade de Brasília (Ceilândia).O trabalho desenvolvido buscou sistematizar processos experimentados em cada um dos cursos analisados, resgatando os antecedentes, a criação e a implantação, revisando documentação de etapas anteriores e atuais sobre os cursos em questão (Belisário et al., 2013).

Um primeiro aspecto que merece destaque é a não uniformidade em relação às denominações.Dosnovecursosanalisados, seisforamintituladosgraduaçãoem saúdecoletiva, dois apresentaram-se como graduação em sistemas e serviços de saúde e um como análise de políticas e sistemas de saúde. Todos ressaltaram a importância da formação de profissionais voltados para a gestão e gerência dos serviços de saúde, em especial dos integrantes do SUS.

\footnotetext{
1 O projeto Análise da Implantação dos Cursos de Graduação em Saúde Coletiva em Diferentes Regiões do Brasil, desenvolvido pela Abrasco por meio do seu GT de Trabalho e Educação na Saúde, contou com o apoio da Opas e da Secretaria de Gestão do Trabalho e da Educação na Saúde do Ministério da Saúde (SGTES/MS).
} 
Contexto político-institucional de criação dos cursos nas instituições de ensino superior

A maioria dos cursos apresentou como justificativa de criação preencher lacuna no mercado das profissões de saúdeno campo da gestão dos serviços esistemas, com domínio de conhecimentos em epidemiologia, planejamento, programação físico-financeira, avaliação, vigilância da saúde e promoção da saúde. Eles reconheciam a ausência de profissionais graduados aptos para tal função ea necessidade de profissionalização dos quadros degestão.

Em relação à finalidade do curso, os projetos foram unânimes quando preconizavam formar profissionais sanitaristas para a atuação no SUS. Consideravam que o perfil do egresso deveria estar condizente com as diretrizes curriculares nacionais (DCNs) para os cursos da área da saúde: formar profissionais com visão humanista e crítico-reflexiva, entre outras características; desenvolver trabalho em equipe; atuar na articulação com outros campos do saber; atuar no campo da gestão em saúde (planejamento, avaliação, sistemas de informação), no das práticas de promoção, vigilância, prevenção e ações educativas e na intersetorialidade.

Foram registradas, com menor frequência, outras características no perfil desejado: ser profissional generalista com visão interdisciplinar, sanitarista, auditor, assessor ou consultor em saúde; ser pesquisador com domínio de estatística e informática; atuar na saúde ocupacional com domínio em riscos e ocupações; ter capacidade de liderança, articulação e negociação política e organização do processo de trabalho nas instituições de saúde; atuar na formulação de estratégias de intervenção nos diferentes modelos de atenção à saúde; ser indutor da saúde; atuar na saúde e não na doença; diagnosticar problemas coletivos de saúde e intervir neles; prestar primeiros socorros às vítimas de acidentes ou de mal súbito; atuar na formação e capacitação de recursos humanos; dominar a legislação em saúde e praticar a arte da análise institucional.

No que tange ao processo de criação do curso, o Reuni foi o fator mencionado nos PPPs que mais influenciou a abertura dos cursos, embora tenha sido lembrado também o fato de já existir um processo de discussão sobre o ensino de graduação em SC. No que se refere ao curso ou departamento quelideroua criaçãoe implantação, a maioria destacou os institutos ou departamentos de SC. Tal criação foi possível principalmente pelas parcerias internas, mais do que pelas externas. A adesão foi constatada em todos os PPPs analisados, apenas em um deles ela não apareceu explicitamente citada - mas, verificando-se as disciplinas ofertadas, constatou-se a participação de cursos de diferentes áreas no currículo proposto. As parcerias mais citadas foram das seguintes áreas: ciências econômicas, ciências da educação, ciências da saúde, ciências biomédicas e biológicas. Apenas três PPPs explicitaram o envolvimento dos gestores nas discussões sobre a criação e o desenvolvimento dos cursos. No entanto, foram mencionadas iniciativas prévias de articulação ensino-serviço e parcerias com as secretarias municipais e estaduais de saúde nos processos de ensino de graduação e pósgraduação (Belisário et al., 2013). 
Por último, no que se refere às condições que facilitaram ou dificultaram a criação do curso, destacam-se, no primeiro caso, o Reuni, seguido do Pró-Saúde, como fatores facilitadores. As dificuldades não são apresentadas explicitamente e sim nas entrelinhas, podendo-se perceber a questão da quantidade equalificação do corpo docente para o ensino da saúde coletiva no âmbito da graduação.

\section{Componentes curriculares}

A segunda dimensão analisada incluiu a orientação do projeto pedagógico, segundo a qual todos os cursos tinham seus desenhos específicos preservando o foco em administração, gerência, epidemiologia, vigilância da saúde, promoção da saúde, política, planejamento e gestão em saúde. Os PPPs explicitavam o compromisso com a formação centrada no aluno e, na sequência, com o currículo estruturado por competências; processo ensino-aprendizagem baseadoem projetoseemresoluçãodeproblemas;aprendizagemorientadapelaação-reflexãoação; aprendizagem pró-ativa e ação interrogadora; currículo integrado; indissociabilidade entre ensino-pesquisa e extensão; metodologias ativas e diversificação das atividades acadêmicas quanto ao tipo e natureza. Pontualmente, houve PPPs que distinguiam também o ensino a distância de maneira complementar; o ensino tutorial; atividades integradas com momentos de concentração e dispersão; presença de orientador acadêmico desde o ingresso no curso; orientador específico para o desenvolvimento do trabalho de conclusão do curso (TCC); e, por fim, o currículo baseado na articulação entre teoria e prática.

Comocenários de práticas, foram propostosnosPPPs:a existência deespaços na própria universidade, tais como laboratórios, bibliotecas e salas de aula; serviços de saúde, tais como os hospitais públicos e privados, centros/unidades locais de saúde, policlínicas, unidades de urgência e emergência; organizações e espaços não governamentais; saúde suplementare unidades deadministraçãocentral,comosecretariasmunicipaiseestaduais de saúde. De forma singular, foi citado o Ministério da Saúde como um dos possíveis cenários de aprendizagem, assim como a articulação com espaços educativos do SUS e as unidades de saúde inseridas na área programática da universidade.

Sobreas competências ehabilidades gerais definidas nosPPPs, predominaramaquelas relacionadas ao perfil de um administrador/gestor e as relacionadas às DCNs dos cursos de saúde, tais como organização da atenção à saúde, tomada de decisão, liderança, administração e gerenciamento. Promover a educação permanente e a saúde e ter o perfil de um pesquisador em SC também foram observados na maioria dos PPPs. Indicaram-se ainda as atuações do futuro profissional no campo da saúde coletiva, no sistema de saúde, no trabalho em SC, assim como em informação de saúde e com grupos populacionais.

No que se refere às competências e habilidades específicas, foram citados, entre outras: atuação nos determinantes do processo saúde-doença; domínio das ferramentas de 
gestão (planejamento, programação, avaliação, auditoria e regulação); educação permanente; uso da epidemiologia e da estatística na organização dos serviços e sistemas de saúde; atuação na mudança dos modelos de atenção e redes de atenção; vigilância à saúde; gestão do trabalho; gestão da educação na saúde; processo de trabalho em saúde; negociação deconflitos;informação e comunicação em saúde para a gestão eformulação, implementação e avaliação de políticas públicas. Apenas um dos PPPs citou o domínio de conhecimentos sobre a legislação em saúde.

Em relação à organização curricular, embora tenha predominado o eixo relativo ao campo da SC com conteúdos de epidemiologia, ciências sociais em saúde e políticas, planejamento, gestão e avaliação em saúde, cada curso apresentou sua especificidade, com destaque para os principais eixos:

- Curso I: economia; gestão de recursos humanos; gestão financeira; saúde coletiva.

- Curso II: saúde e sociedade; educação e comunicação em saúde; estudo morfofuncional humano; estatística e informática em saúde; saúde coletiva (epidemiologia, vigilância, promoção da saúde, política, planejamento e gestão em saúde).

- Curso III: políticas públicas e sistemas de saúde; planejamento, gestão e avaliação; saúde, sociedade e humanidades; análise de situação e vigilância à saúde; pesquisa e bioestatística; promoção e educação da saúde.

- Curso IV: saúde coletiva; ciências biológicas e saúde coletiva; saúde e sociedade; epidemiologia;métodos de investigação; planejamentoegestão; produção, ambiente e saúde; vigilância da saúde.

- CursoV: saúde e sistemas de saúde; planejamento e organização das ações e serviços de saúde; gestão em saúde.

- CursoVl:mododevida;sistemas biológicos;especificidades dofazerem saúdecoletiva; cenários de práticas em saúde coletiva.

- Curso VII: ciências sociais básicas e aplicadas em saúde coletiva; ciências da vida e tecnológicas em saúde; ciências da saúde coletiva; conteúdos e atividades integradores.

- Curso VIII: fundamentos de ciências humanas e biológicas em saúde; saúde e sociedade; política, planejamento e gestão em saúde; epidemiologia e vigilância em saúde; pesquisa em saúde e eixo integrador.

- Curso IX: saúde coletiva; humanidades; saúde e sociedade; política, planejamento e gestão em saúde; epidemiologia e vigilância em saúde.

As estratégias de avaliação do processo ensino-aprendizagem mais citadas nos PPPs foram: avaliação formativa e somativa processual e contínua; trabalhos integrativos de avaliação; fóruns avaliativos; avaliação quantitativa e qualitativa; TCC e avaliação docente. 


\section{Oferta de cursos}

Nessa dimensão investigada observou-se o predomínio do nome do curso graduação em saúde coletiva (seis). Todos os cursos encontravam-se na modalidade bacharelado, predominantemente no regime de oferta anual, com uma e com duas entradas. O número de vagas no vestibular variou de trinta a cem vagas/ano, com uma relação candidato/vaga de 3,5, em média. A maioria disponibilizava seus cursos no turno de funcionamento noturno; dois deles eram diurnos e um em período integral. A carga horária total estava dentro das normas estabelecidas para os cursos de graduação, variando de 2.780 a 5.480 horas, seguindo o padrão dos cursos já existentes. Sobre a relação carga horária teórica/prática, predominava a parte teórica, embora com uma preocupação em relaçãoà prática concentrada principalmente nos últimos anos do curso. Apenas um curso informou ter uma carga horária prática maior que a teórica. A unidade acadêmica mais citada como sede do curso foram os centros de ciências da saúde, com destaque para a enfermagem. Também foram mencionados os institutos de saúde coletiva por dois cursos e a faculdade de medicina por um deles.

Sobre corpo docente e infraestrutura disponível, da maioria dos projetos constavam a quantidade, a titulação e o regime de trabalho dos professores, sendo eles bastante heterogêneos entresi.Observou-sea presença de docentes de outros cursos edepartamentos parceiros na maioria das propostas, profissionais de serviços que poderiam atuar como orientadores temáticos e docentes a serem contratados por meio do Reuni para compor o quadro fixo dos cursos.

\section{Repercussões sobre o Campo da Saúde Coletiva e sobre a Abrasco}

A referida pesquisa resultou em uma série de desdobramentos dentro da Abrasco, entre eles a realização de oficinas de trabalho, coordenadas pelo GT de Trabalho e Educação na Saúde, discutindo temas identificados como relevantes, como mercado de trabalho, capacitação dos docentes e novos cenários de aprendizagem, entre outros. O Fórum de Graduação em Saúde Coletiva, formalmente instalado durante o $1^{\circ}$ Congresso de Política, Planejamento e Gestão, em 2010, representou outro ator para a implementação dos CGSC. Legitimou no interior da Abrasco a graduação em SC e favoreceu o processo organizativo e político dos cursos. A coordenação era exercida de forma colegiada por três docentes, estimulando discussões importantes sobre diversos temas, entre eles, diretrizes curriculares, referencial de curso, inserção no mercado de trabalho e regulamentação da profissão. Desse modo, a Abrasco participou do grande desafio da formação de novos sanitaristas e da sua incorporação no SUS, reafirmando a sua tradição de protagonista na construção da RSB.

A criação dos CGSC vem-se constituindo numa oportunidade para refletir sobre o campo da SC, bem como suas convergências e distinções com a saúde pública. Do mesmo modo, possibilitou uma discussão sobre a RSB, a reorientação das políticas públicas e o 
desenvolvimento do SUS (Bosi \& Paim, 2010). Propiciou ainda "revisitar os marcos do campo e, principalmente, projetar a inserção deste 'novo' sanitarista nesta arena política" (Ruela, 2013:22), assim como refletir sobre a identidade desses profissionais orgânica aos valores e princípios da RSB. Como se alertou em outra oportunidade:

A experiência e a literatura ensinam: o elemento que confere identidade própria ao profissional, essencial na constituição de uma carreira, em especial noâmbito da profissionalização em saúde, não é dado pela especialização. Constrói-se no longo e complexo processo de socialização de saberes - formais e tácitos - sintetizados na experiência de se graduar em uma dada área efazer parte de um segmento profissional, seja profissão sociologicamente estabelecida ou ainda em processo. Não por acaso as graduações (e não as pós-graduações) vêm sendo sistematicamente examinadas como base de constituição do que a Sociologia das Profissões considera"profissão". (Bosi \& Paim, 2010: 2.033)

Em 2014, já havia 21 cursos implantados e profissionais trabalhando ou disponíveis para ingresso no SUS. A inserção desses novos profissionais no mercado de trabalho produziu um intenso debate nos meios acadêmicos, nos serviços de saúde, na Abrasco e nas instâncias colegiadas dos gestores do SUS diante dos desafios e dificuldades enfrentados pelos egressos dos CGSC, com movimentos de apoio e resistência à nova profissão.

\section{Desafios da Prática, do Mercado de Trabalho e das Políticas de Saúde}

Admite-se que o mercado de trabalho para os agentes das práticas de SC não seria algo dado a priori, mas sim o resultado sempre dinâmico da correlação de forças políticas e ideológicas que defendem projetos como os da RSB e doSUS ou se opõem a eles. Desse modo, incluem-se na agenda política questões como a regulamentação da profissão, a necessidade de um conselho corporativo para a categoria, a abertura de postos de trabalho, o acolhimento aos novos sanitaristas e os planos de cargos e carreiras (Paim \& Pinto, 2013).

Em resposta aos que questionam a existência de um mercado, tem sido destacada a participação do corpo discente e dos alunos como protagonistas na criação da carreira e no avanço do processo de profissionalização (Bosi \& Paim, 2010). Esse protagonismo articula-se com os esforços de consolidação do SUS, cuja estabilidade requer profissionais com perfis equivalentes aos propostos pelos CGSC, comprometidos com a diretriz da integralidade da atenção e com os esforços de redefinição das práticas de saúde. A portaria n. 256, de 11 de março de 2013, da Secretaria de Atenção à Saúde do Ministério da Saúde (Brasil, 2013), por exemplo, ao estabelecer novas regras para cadastramento dos Núcleos de Apoio à Saúde da Família (Nasf), inclui no seu art. $5^{\circ}$ o sanitarista como profissional graduado em saúde coletiva (Ruela, 2013).

No que diz respeito às práticas de saúde e à natureza do processo de trabalho em SC, os cursos precisam ultrapassar nos seus PPPs e na sua prática cotidiana a visão de necessidades de saúde como "objeto ingênuo" (Donnangelo, 1976: 24). A reflexão crítica 
sobre a emergência e o desenvolvimento da SC no Brasil, em contraposição à saúde pública institucionalizada, pode estabelecer um vínculo entre um saber renovado e práticas políticas diferenciadas, criticando o sanitarismo dominante e suas práticas ausentes ou equivalentes. Portanto, os cursos podem optar pela reprodução da saúde pública institucionalizada e, ainda, pela restauração da saúde pública convencional de matriz flexner-rockefelleriana ou ousar inovar, mesmo diante de contradições e da ideologia neoliberal, como mostram os primeiros estudos sobre a sua concepção e implementação (Paim, 2006; Bosi \& Paim, 2010; Paim \& Pinto, 2013; Ruela, 2013; Santos, 2014).

É possível que certos cursos já estejam problematizando essas matrizes, contradiçõese ideologias ou realizando um mapeamento de serviços e instituições que reproduzem a saúde pública institucionalizada, nem sempre orgânica ao desenvolvimento do SUS. Tais reflexões e debates tendem a gerar novas questões para a graduação em SC, explorando diferentes possibilidades de prática.

A constituição de novos sujeitos da SC, nessa perspectiva, supõe a articulação da prática teórica e da prática política, bem como o desenvolvimento da atitude crítica e de uma aptidão crítica (Testa, 2007). Esse caminho pode ajudar a superar uma consciência mágica ou ingênua dos graduandos, desenvolvendo uma consciência crítica que os estimule a empreenderum pensamentoe umagir estratégicos, seja na análise política, seja na formulação e implementação de políticas de saúde (Paim \& Pinto, 2013).

\section{Comentários Finais}

Este capítulo, apoiado nos primeiros estudos, pesquisas, dissertações e teses que tomaram como objeto os CGSC, procurou mostrar a participação da Abrasco na construção dessa nova modalidade de constituição de sujeitos das práticas de saúde, assim como destacar certas possibilidades que se abrem diante do processo de implementação da graduação em SC no Brasil.

Contudo, a Abrasco pode ir além da discussão de aspectos históricos, conceituais, teóricos, epistemológicos, pedagógicos, metodológicos, técnicos e operacionais da SC na graduação. Um dos seus desafios é contribuir para a constituição de"novos sanitaristas para uma nova sociedade" e indicar "novas faces para o sanitarista", como problematiza Ruela (2013). Esse autor, ao analisar a financeirização da economia, a reestruturação produtiva do capital e a chamada terceira via no Brasil, conclui que"é nessa conjuntura do ensino superior no Brasil que está inserida a proposta dos CGSC que, se não está totalmente afinada com os preceitos da mercadificação do campo, certamente não passará impune a este processo" (Ruela, 2013: 81).

Ainda que determinantes estruturais de sociedades capitalistas induzam à formação de intelectuais orgânicos ao capital, é possível apostarnum processo contra-hegemônico que 
facilite a constituição de novos sujeitos críticos para a SC, a RSB e o SUS (Santos, 2014). Assim, diversos sujeitos podem se mover nessa conjuntura, e a resultante política do seu pensar e agir, progressista ou conservadora, dependerá da correlação de forças e da produção de novos fatos políticos e institucionais. Essa aposta na formação de novos sujeitos e numa práxis contra-hegemônica pode encontrar seus fundamentos e estratégias na experiência acumulada da SC e do processo da RSB.

Os jovens profissionais graduados em SC possivelmente demarcarão suas trajetórias delineandosuaidentidadenoestudo, notrabalhoenaslutas paraseremnovossujeitos-agentes das práticas de saúde (Bosi \& Paim, 2010). Como militantes, profissionais e associados da Abrasco, construirão a sua história para além do que é possível prever.

\section{REFERÊNCIAS}

ALMEIDA, M. A área é multiprofissional e interdisciplinar. Radis, 13: 22, 2003.

ASSOCIAÇÃO BRASILEIRA DE SAÚDE COLETIVA (ABRASCO). Relatório final da 3 a Reunião Nacional de Docentes de Medicina Preventiva e Social. Ensino da Saúde Pública, Medicina Preventiva e Social no Brasil, 3: 171-187, 1984.

ASSOCIAÇÃO BRASILEIRA DE SAÚDE COLETIVA (ABRASCO). Relatório de pesquisa análise da implantação dos cursos de graduação em saúde coletiva em diferentes regiões do Brasil. Relatório de Pesquisa. Rio de Janeiro: Abrasco, 2010.

ASOCIACIÓN LATINOAMERICANA Y DEL CARIBE DE EDUCACIÓN EN SALUD PÚBLICA (ALAESP). I Conferencia Panamericana de Educación en Salud Pública. XVI Conferencia de Alaesp. Informe Final. Rio de Janeiro: Ensp, 1994.

BELISÁRIO, S. A. As múltiplas faces do médico-sanitarista. In: MACHADO, M. H. (Org.). Profissões de Saúde: uma abordagem sociológica. Rio de Janeiro: Editora Fiocruz, 1995.

BELISÁRIO, S. A. et al. Implantação do curso de graduação em saúde coletiva: a visão dos coordenadores. Ciência \& Saúde Coletiva, 18(6): 1.625-1.634, 2013.

BOSI, M. L. M. \& PAIM, J. S. Graduação em saúde coletiva: subsídios para um debate necessário. Editorial. Cadernos de Saúde Pública, 25(2): 236-237, 2009.

BOSI, M. L. M. \& PAIM, J. S. Graduação em saúde coletiva: limites e possibilidades como estratégia de formação profissional. Ciência \& Saúde Coletiva, 15(4): 2.029-2.038, 2010.

BOSI, M. L. M.; RAGGIO, R. \& MEDRONHO, R. A. Reflexões em torno de uma profissionalização em saúde pública (Lilacs). Cadernos Saúde Coletiva, 9(1): 5-8, 2001.

BRASIL. Decreto n. 6.096, de 24 abr. 2007. Institui o Programa de Apoio a Planos de Reestruturação e Expansão das Universidades Federais - Reuni. Disponível em:<www.planalto.gov.br/ccivil_03/_ato20072010/2007/decreto/d6096.htm>. Acesso em: 7 abr. 2009.

BRASIL. Ministério da Saúde. Secretaria de Atenção à Saúde. Portaria n. 256, de 11 mar. 2013. Estabelece normas para o cadastramento dos Núcleos de Apoio à Saúde da Família (Nasf). Disponível em: <http:// bvsms.saude.gov.br/bvs/saudelegis/sas/2013/prt0256_11_03_2013.html>. Acesso em: mar. 2015.

CECCIM, R. B. Inovação na preparação de profissionais de saúde e a novidade da graduação na área de saúde coletiva. Boletim da Saúde, 16(1): 9-36, 2002. 
DONNÂNGELO, M. C. F. A pesquisa na área da saúde coletiva no Brasil: a década de 70. Ensino da Saúde Pública, Medicina Preventiva e Social no Brasil, 2: 19-35, 1983.

ELIAS, P. E. Graduação em saúde coletiva: notas para reflexões. Interface - Comunicação, Saúde, Educação, 7(13): 167-170, 2003.

INSTITUTO DE SAÚDE COLETIVA/UNIVERSIDADE FEDERAL DA BAHIA(ISC/UFBA). Relatório do Seminário Graduação em Saúde Coletiva: pertinência e possibilidades. Salvador, 2002.

MACEDO, C. G. El contexto. In: ORGANIZACIÓN PANAMERICANA DE LA SALUD (OPS). La Crisis de la Salud Pública: reflexiones para el debate. Washington: OPS, 1992. (Publicación científica, 540)

PAN AMERICAN HEALTH ORGANIZATION (PAHO). The Crisis of Public Health: reflections for the debate. Washington: Pan American Health Organization, 1992. (Scientific publication, 540)

PADILHA, H. Saúde Pública: campo para profissionalizar em nível de graduação? Salvador, ago. 1997. (Texto elaborado para discutir o tema no ISC/Ufba).

PAIM, J. S. O SUS tem pressa para reorientar modelo. Radis, 13: 23, 2003.

PAIM, J. S. Desafios para a Saúde Coletiva no Século XXI. Salvador: Edufba, 2006.

PAIM, J. S. \& PINTO, I. C. M. Graduação em saúde coletiva: conquistas e passos para além do sanitarismo. RevistaTempus: Actas de SaúdeColetiva, 7(3), 2013. Disponível em:<www.tempusactas.unb.br/index.php/ tempus/article/view/1390>. Acesso em: fev. 2015.

RUELA, H. C. G. A Formação de Sanitaristas e os Cursos de Graduação em Saúde Coletiva no Brasil, 2013. Dissertação de Mestrado Profissional em Saúde, Rio de Janeiro: Escola Politécnica de Saúde Joaquim Venâncio/Fiocruz.

SANTOS, L. Educação e Trabalho na Saúde Coletiva Brasileira: estudo de caso sobre a criação dos cursos de graduaçãonaárea desaúdecoletiva noscenários nacional elocal,2014.TesedeDoutorado, Salvador:Instituto de Saúde Coletiva, Universidade Federal da Bahia.

TEIXEIRA, C. Graduação em saúde coletiva: antecipando a formação do sanitarista. Interface:Comunicação, Saúde, Educação, 16, supl.1: 284-312, 2003.

TESTA, M. Decidir en salud: ¿Quén?, ¿Cómo? y ¿Porqué? Salud Colectiva, 3(3): 247-257, 2007. 